B.Y. CHEN

KODAI MATH. J.

14 (1991), 341-349

\title{
LINEARLY INDEPENDENT, ORTHOGONAL AND EQUIVARIANT IMMERSIONS
}

\author{
BY BANG-YEN CHEN
}

\begin{abstract}
In this article we define the notions of linearly independent and orthogonal immersions and introduce the notion of adjoint hyperquadrics of linearly independent immersions. We investigate the relations between linearly indedendent immersions, orthogonal immersions, equivariant immersions and adjoint hyperquadrics. Several results in this respect are obtained.
\end{abstract}

\section{Introduction}

Let $x: M \rightarrow E^{m}$ be an immersion from an $n$-dimensional, connected manifold $M$ into the Euclidean $m$-space $E^{m}$. With respect to the Riemannian metric $g$ on $M$ induced from the Euclidean metric of the ambient space $E^{m}, M$ is a Riemannian manifold. Denote by $\Delta$ the Laplacian operator of the Riemannian manifold $(M, g)$. The immersion $x$ is said to be of finite type (cf. [1,2] for details) if each component of the position vector field of $M$ in $E^{m}$, also denoted by $x$, can be written as a finite sum of eigenfunctions of the Laplacian operator, that is, if

$$
x=c+x_{1}+x_{2}+\cdots+x_{k}
$$

where $c$ is a constant vector, $x_{1}, \cdots, x_{k}$ are non-constant maps satisfying $\Delta x_{2}$ $=\lambda_{2} x_{2}, i=1, \cdots, k$. If in particular all eigenvalues $\left\{\lambda_{1}, \cdots, \lambda_{k}\right\}$ are mutually different, then the immersion $x$ (or the submanifold $M$ ) is said to be of $k$-type and the decomposition (1.1) is called the spectral decomposition of the immersion $x$.

For a finite type immersion whose spectral decomposition is given by (1.1) we shall always assume in this article that the eigenvalues $\left\{\lambda_{1}, \cdots, \lambda_{k}\right\}$ satisfy $\lambda_{1}<\cdots<\lambda_{k}$ for simplicity. Moreover, for a such immersion we shall choose the Euclidean coordinate system $\left(u_{1}, \cdots, u_{m}\right)$ on $E^{m}$ in such way that $c$ is its origin. Therefore, with respect to the Euclidean coordinate system so chosen, the spectral decomposition of $x$ is given by

Received November 13, 1990. 


$$
x=x_{1}+\cdots+x_{k}, \quad \Delta x_{i}=\lambda_{2} x_{\imath}, \quad i=1, \cdots, k, \lambda_{1}<\cdots<\lambda_{k} .
$$

For a $k$-type immersion $x: M \rightarrow E^{m}$, let $E_{\imath},(i \in\{1, \cdots, k\})$ denote the subspace of $E^{m}$ spanned by $x_{\imath}$. The immersion $x$ is said to be linearly independent if the subspaces $E_{1}, \cdots, E_{k}$ are linearly independent (cf. Definition 2.1 for details). And the immersion $x$ is said to be orthogonal if the subspaces $E_{1}, \cdots, E_{k}$ are mutually orthogonal. Clearly, every orthogonal immersion is a linearly independent immersion. There exist ample examples of orthogonal immersions and ample examples of linearly independent immersions which are not orthogonal.

For each linearly independent immersion $x: M \rightarrow E^{m}$ and each point $p \in M$ we introduce in this paper the notion of the adjoint hyperquadric $Q_{p}$ at $p$. If the submanifold $M$ lies in an adjoint hyperquadric $Q_{p}$ for some point $p \in M$, then all of the adjoint hyperquadrics $Q_{p}, p \in M$ give a common adjoint hyperquadric, denoted by $Q$. The unique adjoint hyperquadric $Q$ so defined is called the adjoint hyperquadric of the linearly independent immersion $x$.

The main purpose of this paper is to introduce the notions of linearly independent and orthogonal immersions and the notion of adjoint hyperquadric of a linearly independent immersion and to investigate the relations between linearly independent immersions, orthogonal immersions, equivariant immersions, and their adjoint hyperquadrics. Several fundamental results in this respect are obtained.

\section{Adjoint hyperquadrics and linearly independent immersions}

Let $x: M \rightarrow E^{m}$ be an immersion of $k$-type and let

$$
x=c+x_{1}+\cdots+x_{k}, \quad \Delta x_{\imath}=\lambda_{2} x_{\imath}, \quad \lambda_{1}<\cdots<\lambda_{k}
$$

be the spectral decomposition of the immersion $x$, where $c$ is a constant vector and $x_{1}, \cdots, x_{k}$ are non-constant maps. For each $i \in\{1, \cdots, k\}$ we put $E_{\imath}=$ Span $\left\{x_{i}(p): p \in M\right\}$. Then each $E_{\imath}$ is a linear subspace of $E^{m}$.

We give the following

DEFINITION 2.1. Let $x: M \rightarrow E^{m}$ be an immersion of $k$-type whose spectral decomposition is given by (2.1). Then the immersion $x$ is said to be linearly independent if the subspaces $E_{1}, \cdots, E_{k}$ are linearly independent, that is, the dimension of subspace spanned by vectors in $E_{1} \cup \cdots \cup E_{k}$ is equal to $\operatorname{dim} E_{1}+$ $\cdots+\operatorname{dim} E_{k}$. And the immersion $x$ is said to be orthogonal if the subspaces $E_{1}, \cdots, E_{k}$ are mutually orthogonal in $E^{m}$.

Obviously, every 1-type immersion is an orthogonal immersion and hence a linearly independent immersion.

Let $x: M \rightarrow E^{m}$ be an immersion of $k$-type whose spectral decomposition is given by (2.1). We choose the Euclidean coordinate system $\left(u_{1}, \cdots, u_{m}\right)$ on $E^{m}$ 
with $c$ as the origin. Then we have the following spectral decomposition of $x$ :

$$
x=x_{1}+\cdots+x_{k}, \quad \Delta x_{\imath}=\lambda_{2} x_{\imath}, \quad \lambda_{1}<\cdots<\lambda_{k} .
$$

For each $i \in\{1, \cdots, k\}$ we choose a basis $\left\{c_{\imath}: j=1, \cdots, m_{\imath}\right\}$ of $E_{\imath}$, where $m_{\imath}$ is the dimension of $E_{\imath}$. Put $l=m_{1}+\cdots+m_{k}$ and let $E^{l}$ denote the subspace of $E^{m}$ spanned by $E_{1}, \cdots, E_{k}$. If the immersion $x$ is linearly independent, then the vectors $\left\{c_{\imath}: i=1, \cdots, k ; j=1, \cdots, m_{j}\right\}$ are linearly independent $l$ vectors in $E^{l}$. Furthermore, we choose the Euclidean coordinate system $\left(u_{1}, \cdots, u_{m}\right)$ on $E^{m}$ such that $E^{\imath}$ is defined by $u_{l+1}=\cdots=u_{m}=0$. Regard each $c_{\imath}$ as a column $l$-vector. We put

$$
S=\left(c_{11}, \cdots, c_{1 m_{1}}, \cdots, c_{k 1}, \cdots, c_{k m_{k}}\right) .
$$

Then the matrix $S$ is a nonsingular $l \times l$ matrix. Let $D$ denote the diagonal $l \times l$ matrix given by

$$
D=\operatorname{Diag}\left(\lambda_{1}, \cdots, \lambda_{1}, \cdots, \lambda_{k}, \cdots, \lambda_{k}\right) \text {, }
$$

where $\lambda_{\imath}$ repeats $m_{i}$-times. If we put $A=S D S^{-1}$, then $A c_{\imath \jmath}=\lambda_{i} c_{\imath}$, for any $i \in$ $\{1, \cdots, k\}$ and $\jmath \in\left\{1, \cdots, m_{i}\right\}$. Therefore, we have

$$
\Delta x=A x
$$

for the immersion $x: M \rightarrow E^{l}$ induced from the original immersion $x: M \rightarrow E^{m}$. By regarding the $l \times l$ matrix $A$ as an $m \times m$ matrix in a natural way (with zeros for each of the additional entries), we obtain

$$
\Delta x=A x, \quad A=\left(a_{\imath}\right)
$$

for the immersion $x: M \rightarrow E^{m}$.

If $x: M \rightarrow E^{m}$ is a minimal immersion, then $A=0$. If $x: M \rightarrow E^{l}\left(\subset E^{m}\right)$ is a non-minimal full immersion, then the Euclidean coordinate functions $u_{1}, \cdots, u_{l}$ of $E^{l}$, restricted to $M$, do not satisfy any linear equation. Thus, the coordinate functions $\left.u_{1}\right|_{M}, \cdots,\left.u_{l}\right|_{M}$ of $M$ in $E^{l}$ are linearly independent functions. Therefore, if $B$ is any $l \times l$ matrix such that $\Delta x=B x$, then $A=B$. Hence, the $l \times l$ matrix $A$ in $(2.5)^{\prime}$ defined above is unique. Consequently, if the (original) immersion $x: M \rightarrow E^{m}$ is a non-minimal, linearly independent immersion, then the $m \times m$ matrix $A$ given in (2.5) is also uniquely defined (with respect to the Eucli- dean coordinate system so chosen).

By using this $m \times m$ matrix $A$ in (2.5) defined above, we give the following

DEFinition 2.2. Let $x: M \rightarrow E^{m}$ be a non-minimal, linearly independent immersion whose spectral decomposition is given by $(2.1)$. Let $u=\left(u_{1}, \cdots, u_{m}\right)$ be a Euclidean coordinate system on $E^{m}$ with $c$ as its origin and let $A$ be the $m \times m$ matrix in (2.5) associated with the immersion $x$ defined above. Then, for any point $p \in M$, the equation 


$$
\langle A u, u\rangle:=\sum_{i, j}^{m} a_{i j} u_{i} u_{j}=c_{p}, \quad\left(c_{p}=\langle A x, x\rangle(p)\right)
$$

defines a hyperquadric $Q_{p}$ in $E^{m}$. We call the hyperquadric $Q_{p}$ the adjoint hyperquadric of the immersion $x$ at $p$. In particular, if $x(M)$ is contained in an adjoint hyperquadric $Q_{p}$ of $x$ for some point $p \in M$, then all of the adjoint hyperquadrics $\left\{Q_{p}: p \in M\right\}$ give a common adjoint hyperquadric, denoted by $Q$. We call the hyperquadric $Q$ the adjoint hyperquadric of the linearly independent immersion $x$.

The following result gives a necessary and sufficient condition for a compact, linearly independent submanifold to lie in its adjoint hyperquadric.

THEOREM 2.1. Let $x: M \rightarrow E^{m}$ be a linearly independent immersion from a compact manifold $M$ into $E^{m}$ whose spectral decomposition is given by (2.2). Then $M$ is immersed into the adjoint hyperquadric of $x$ if and only if $M$ is immersed into a hypersphere of $E^{m}$ centered at the origin.

Proof. Assume that $M$ is immersed into a hypersphere $S^{m-1}(r)$ of $E^{m}$ with radius $r$ centered at the origin. Denote by $H$ and $\bar{H}$ the mean curvature vectors of $M$ in $E^{m}$ and of $M$ in $S^{m-1}(r)$, respectively. Then we have

$$
H=\bar{H}-\frac{1}{r} x \text {. }
$$

This implies $\langle H, x\rangle=-r$. Since $\Delta x=-n H, n=\operatorname{dim} M$, we obtain $\langle\Delta x, x\rangle=n r$. Therefore, by (2.5), we conclude that $M$ is immersed into the adjoint hyperquadric defined by $\langle A u, u\rangle=n r$, where $n r$ is a constant.

Conversely, suppose that $x: M \rightarrow E^{m}$ is a linearly independent immersion of a compact manifold $M$ such that $x(M)$ is contained in an adjoint hyperquadric $Q_{p}$ for some point $p$. Then we have $\langle A x, x\rangle=c_{p}$ where $c_{p}$ is the constant given by $c_{p}=\langle A x, x\rangle(p)$. Since $A x=\Delta x=-n H$, we have

$$
\langle n H, x\rangle=-c_{p} \text {. }
$$

Because $M$ is compact, we also have (cf. [1, p. 193])

$$
\int_{M}\{1+\langle H, x\rangle\} * 1=0 \text {. }
$$

Formulas (2.8) and (2.9) imply $c_{p}=-n$. Therefore,

$$
\Delta\langle x, x\rangle=2\langle\Delta x, x\rangle-2 n=-2 n(\langle H, x\rangle+1)=0 .
$$

Thus, by Hopf's lemma, $\langle x, x\rangle$ is a constant. Hence $M$ is immersed into a hypersphere of $E^{m}$ centered at the origin. This completes the proof of the theorem. 
Remark 2.1. Although the implication $(\Leftarrow)$ in Theorem 2.1 holds in general without the assumption of compactness, the implication $\Rightarrow$ does not hold in general if $M$ is not compact. For example, let $M$ be the product surface of a unit plane circle and a line. Then the inclusion map $x$ of $M$ into $E^{3}$ defined by

$$
x(u, v)=(\cos u, \sin u, v)
$$

is a non-spherical, linearly independent immersion whose adjoint hyperquadric is given by $u_{1}^{2}+u_{2}^{2}=1$. It is clear that $M$ coincides with the adjoint hyperquadric $Q$ of $M$ in $E^{3}$.

We give the following lemma for later use.

LEMMA 2.2. Let $x: M \rightarrow E^{m}$ be a linearly independent immersion whose spectral decomposition is given by (2.2). Then, for any vector $X$ tangent to $M$, we have

$$
\tilde{\nabla}_{X} x_{\imath}=X_{\imath}, \quad i=1, \cdots, k,
$$

where $X=X_{1}+\cdots+X_{k}, X_{i} \in E_{\imath}=\operatorname{Span}\left\{x_{\imath}\right\}$.

Proof. This lemma follows from the linearly independence of the immersion and the fact $\tilde{\nabla}_{X} x=X$, where $\tilde{\nabla}$ is the Levi-Civita connection of $E^{m}$.

The following result provides a necessary and sufficient condition for a linearly independent immersion to be an orthogonal immersion in terms of the adjoint hyperquadric.

THEOREM 2.3. Let $x: M \rightarrow E^{m}$ be a non-minimal, linearly independent immersion. Then $M$ is immersed by $x$ as a minimal submanifold of the adjoint hyperquadric if and only if the immersion $x$ is an orthogonal immersion.

Proof. Let $x: M \rightarrow E^{m}$ be a non-minimal, linearly independent immersion whose spectral decomposition is given by (2.2). Then there exists an $m \times m$ matrix $A=\left(a_{\imath}\right)$ such that $\Delta x=A x$ as we mentioned before. Assume that $M$ is immersed as a minimal submanifold of the adjoint hyperquadric $Q$ of $x$ defined by

$$
\sum_{i, j}^{m} a_{\imath j} u_{i} u_{j}=b
$$

for some constant $b$. We put $f=\sum a_{i j} u_{i} u_{\jmath}$. Then the adjoint hyperquadric $Q$ is a level hypersurface of $f$ and the gradient vector field of $f$ is given by

$$
\begin{aligned}
& \nabla f=\left(2 a_{11} u_{1}+\left(a_{12}+a_{21}\right) u_{2}+\cdots+\left(a_{1 m}+a_{m 1}\right) u_{m}, \cdots\right. \\
& \left.\cdots,\left(a_{1 m}+a_{m 1}\right) u_{1}+\cdots+\left(a_{m-11}+a_{1 m-1}\right) u_{m-1}+2 a_{m m} u_{m}\right) .
\end{aligned}
$$

On the other hand, $-n H=\Delta x=A x$ yields 


$$
-n H=\left(a_{11} u_{1}+\cdots+a_{1 m} u_{m}, \cdots, a_{m 1} u_{1}+\cdots+a_{m m} u_{m}\right)
$$

at every point $p \in M$. Since $M$ is assumed to be immersed in the adjoint hyperquadric $Q$ as a minimal submanifold, $\nabla f$ is parallel to the mean curvature vector $H$ of $M$ in $E^{m}$ along the image of $x$. Thus, there is a function $\mu$ on $M$ such that $\nabla f=\mu H$.

If the immersion $x$ is full, then the coordinate functions $u_{1}, \cdots, u_{m}$, restricted to $M$, are linearly independent, since the immersion is nonlinear. Thus, by using (2.11), (2.12) and the condition $\nabla f=\mu H$, we may conclude that $\mu=2$ and $A$ is a symmetric matrix. On the other hand. from (2.2) and (2.5), we have

$$
\left(A x_{1}-\lambda_{1} x_{1}\right)+\cdots+\left(A x_{k}-\lambda_{k} x_{k}\right)=0 .
$$

Because $\Delta\left(A x_{\imath}\right)=A\left(\Delta x_{\imath}\right)=\lambda_{\imath} A x_{\imath}$, equation (2.13) yields

$$
\lambda_{1}^{J}\left(A x_{1}-\lambda_{1} x_{1}\right)+\cdots+\lambda_{k}^{J}\left(A x_{k}-\lambda_{k} x_{k}\right)=0, \quad j=1,2, \cdots, k .
$$

Because $\lambda_{1}<\cdots<\lambda_{k}$, we obtain $A x_{\imath}=\lambda_{\imath} x_{\imath}, i=1, \cdots, k$. Since $A$ is symmetric and distinct eigenspaces of a symmetric matrix are mutually orthogonal, the immersion $x$ is an orthogonal immersion.

If the immersion $x$ is not full, then $x$ gives rise a full, linearly independent immersion $\bar{x}: M \rightarrow E^{l}\left(\subset E^{m}\right)$. Let $\bar{A}$ denote the corresponding $l \times l$ matrix with $\Delta \bar{x}=\bar{A} \bar{x}$. Then $A$ is obtained from $\bar{A}$ by putting zeros on the additional entries as we mentioned before. Since $M$ is immersed by $x$ as a minimal submanifold in the adjoint hyperquadric of $x, M$ is immersed by $\bar{x}$ as a minimal submanifold in the adjoint hyperquadric of $\bar{x}$. Thus, by applying the previous case, we conclude that the immersion $\bar{x}$ is orthogonal and hence the immersion $x$ is also orthogonal.

Conversly, assume $x$ is an orthogonal immersion. Let

$$
f=\lambda_{1}\left\langle x_{1}, x_{1}\right\rangle+\cdots+\lambda_{k}\left\langle x_{k}, x_{k}\right\rangle .
$$

Then Lemma 2.2 implies

$$
X f=2\left\{\lambda_{1}\left\langle x_{1}, X_{1}\right\rangle+\cdots+\lambda_{k}\left\langle x_{k}, X_{k}\right\rangle\right\}=2\langle\Delta x, X\rangle=0
$$

for any vector $X$ tangent to $M$. From this we conclude that $M$ is immersed in an adjoint hyperquadric given by $\langle A u, u\rangle=b$ for some constant $b$ and also the gradient of $f$ is given by $-2 n H$. Since the gradient of $f$ is normal to the hyperquadric, $M$ is immersed in the adjoint hyperquadric as a minimal submanifold. This completes the proof of the theorem.

Remark 2.2. From the definition of linearly independent immersions we see that if $x: M \rightarrow E^{m}$ is a linearly independent $k$-type immersion of an $n$-dimensional manifold $M$ into $E^{m}$, then $k \leqq m$.

Remark 2.3. In [3] the relations between linearly independent immersions, 
orthogonal immersions and the conditions introduced in $[5,6]$ are further dicussed. Also see [7] for a special case of Theorem 2.3.

Remark 2.4. There exist ample examples of orthogonal immersions and ample examples of linearly independent immersions which are not orthogonal. For instances, every $k$-type curve lying fully in $E^{2 k}$ is an example of linearly independent immersion and the immersion of the curve is orthogonal if and only if it is a $W$-curve. Moreover, the product of any two linearly independent immersions is again a linearly independent immersion.

\section{Equivariant immersions and adjoint hyperquadrics}

The purpose of this section is to prove the following general result for equivariant immersions of a compact homogeneous spaces.

THEOREM 3.1. Let $x: M \rightarrow E^{m}$ be an equivariant isometric immersion of a compact $n$-dimensional Riemannian homogeneous space $M$ into $E^{m}$. Then $M$ is immersed as a minimal submanifold of the adjoint hyperquadric.

Proof. Let $x: M \rightarrow E^{m}$ be an equivariant isometric immersion of a compact connected Riemannian homogeneous space $M$ into $E^{m}$. Without loss of generality we may assume the immersion is full. It is known that the immersion $x$ is spherical. Thus $x(M)$ is contained in a hypersphere $S^{m-1}$ of $E^{m}$. Without loss of generality, we may also assume that $S^{m-1}$ is centered at the origin of $E^{m}$. Denote by $G=I_{0}(M)$ the identity component of the group of all isometries of $M$. Then $G$ is a compact Lie group which acts transitively on $M$ and there is a Lie homomorphism $\phi: G \rightarrow S O\left(E^{m}\right)$ such that $x(g(p))=\phi(g)(x(p))$ for every $g \in G$ and $p \in M$. Because $\left(\phi, E^{m}\right)$ is a representation of the compact Lie group $G,\left(\phi, E^{m}\right)$ is the direct sum of some irreducible subrepresentations $\left(\phi_{1}, E_{1}\right), \cdots$, $\left(\phi_{k}, E_{k}\right)$ such that $E^{m}$ is the Euclidean direct sum $E_{1} \oplus \cdots \oplus E_{k}$ of $E_{1}, \cdots, E_{k}$. Let $x_{\imath}$ denote the $E_{\imath}$-component of $x$. Then we have

$$
x_{i}(g(p))=\phi_{i}(g)\left(x_{i}(p)\right), \quad g \in G, p \in M, i=1, \cdots, k .
$$

Since $E_{1}, \cdots, E_{k}$ are mutually orthogonal in $E^{m}, x=x_{1}+\cdots+x_{k}$ is an orthogonal decomposition.

Now we claim that each $x_{2}$ is a 1-type map, that is, $\Delta x_{2}=\lambda_{2} x_{2}, i \in\{1, \cdots, k\}$, for some real numbers $\lambda_{2}$. In order to do so, we choose a fixed point $o \in M$. Denote by $K$ the isotropy subgroup of $G$ at $o$. Then $M$ can be identified with $G / K$ in a natural way. Consider a biinvariant Riemannian metric on the compact Lie group $G$ such that the projection $\pi: G \rightarrow M=G / K$ is a Riemannian submersion. Let $e_{1}, \cdots, e_{N}$ be any orthonormal basis of the Lie algebra $g=T_{e} G$ of $G$, where $e$ is the identity element of $G$.

For each $i \in\{1, \cdots, k\}$, denote also by $\phi_{i}$ the homomorphism $\mathfrak{g} \rightarrow \mathfrak{g}_{\mathfrak{D}}\left(E_{\imath}\right)$ induced from $\phi_{i}: G \rightarrow E_{\imath}$, where $\mathfrak{g} \mathfrak{p}\left(E_{\imath}\right)$ is the Lie algebra of $S O\left(E_{\imath}\right)$. Then each 
$\phi_{i}\left(e_{a}\right)$ is a skew-symmetric linear transformation of $E_{\imath}$ and $\sum_{a=1}^{N} \phi_{i}\left(e_{a}\right)^{2}$ is a symmetric linear transformation. Let $A d: G \rightarrow G L(\mathrm{~g})$ be the adjoint representation of $G$. Since $\operatorname{ad}(g)(h)=g h g^{-1}$ and $\phi_{i}$ is a Lie homomorphism, we have

$$
\phi_{i}(g) \phi_{i}(X) \phi_{i}\left(g^{-1}\right)=\phi_{i}(A d(g) X)
$$

for any $X \in \mathfrak{g}, g \in G$ and $i \in\{1, \cdots, k\}$. Therefore we find

$$
\phi_{i}(g)\left(\sum_{a=1}^{N} \phi_{i}\left(e_{a}\right)^{2}\right) \phi_{i}\left(g^{-1}\right)=\sum_{a=1}^{N} \phi_{i}\left(A d(g) e_{a}\right)^{2}=\sum_{a=1}^{N} \phi_{i}\left(e_{a}\right)^{2}
$$

for any $g \in G$. This shows that $\sum_{a=1}^{N} \phi_{i}\left(e_{a}\right)^{2}$ lies in the centralizer of $\phi_{i}(G)$. Since the representation $\left(\phi_{i}, E_{\imath}\right)$ is irreducible, Schur's lemma in representation theory implies that

$$
\sum_{a=1}^{N} \phi_{i}\left(e_{a}\right)^{2}=-\lambda_{i} I_{\imath}
$$

for some constants $\lambda_{\imath}$, where $I_{\imath}$ is the identity transformation on $E_{\imath}$. On the other hand, it is known that the Laplacian of $x_{\imath}$ is given by (see, for instances, $[9$, pp. 17-21])

$$
\Delta x_{i}(p)=-\left.\sum_{a=1}^{N} \frac{d^{2}}{d t^{2}} x_{i}\left(\exp t e_{a}\right)\right|_{t=0}=-\sum_{a=1}^{N} \phi_{i}\left(e_{a}\right)^{2}\left(x_{i}(p)\right) .
$$

Therefore, by (3.2) and (3.3), we obtain the claim. Consequently, the immersion $x$ is an orthogonal immersion. Hence, by Theorem $2.3, M$ is immersed as a minimal submanifold of the adjoint hyperquadric. This completes the proof of the theorem.

Remark 3.1. The method of proof of Theorem 3.1 is similar to the proof of [8]. For equivariant isometric immersions of compact Riemannian homogeneous spaces with irreducible isotropy actions, see also [4] and [8].

\section{Immersions with $\langle\Delta x, x\rangle=0$.}

From (2.5) and the definition of adjoint hyperquadrics, we see that if a manifold $M$ is immersed by an immersion $x$ as a submanifold of an adjoint hyperquadric at some point $p \in M$, then $\langle\Delta x, x\rangle$ is a constant function on $M$.

In this section we ask the following simple geometric problem.

PROBLEM 4.1. Let $x: M \rightarrow E^{m}$ be an immersion. When the immersion satisfies the condition $\langle\Delta x, x\rangle=0$ ?

Since the position vector $x(p)$ of an $n$-dimensional cone in $E^{m}$ with vertex at the origin of $E^{m}$ is tangent to the cone, we see that every cone in $E^{m}$ with vertex at the origin satisfies the condition $\langle\Delta x, x\rangle=0$. Also $\Delta x=-n H$ implies that every minimal submanifold of $E^{m}$ also satisfies the condition.

The following result shows that in fact cones and minimal submanifolds of $E^{m}$ are the only such submanifolds when the codimension is one. 
Proposition 4.1. Let $x: M \rightarrow E^{n+1}$ be an immersion from an $n$-dimensional manifold $M$ into $E^{n+1}$. Then $\langle\Delta x, x\rangle=0$ identically on $M$ if and only if locally $M$ is immersed either as a minimal hypersurface or as a hypercone with vertex at the origin of $E^{n+1}$.

Proof. Let $x: M \rightarrow E^{n+1}$ be an immersion from an $n$-dimensional manifold $M$ into $E^{n+1}$ satisfying $\langle\Delta x, x\rangle=0$ on $M$. If $x$ is a minimal immersion, there is nothing to prove. So we may assume that the mean curvature vector $H$ of the immersion $x: M \rightarrow E^{n+1}$ is nonzero. Since $\Delta x=-n H$, the condition $\langle\Delta x, x\rangle$ $=0$ implies that the position vector field $x$ of $M$ in $E^{n+1}$ is a tangent vector field of $M$. Let $\gamma(s)$ be any integral curve of $x$ parameterized by the arclength $s$. Then the velocity vector $\gamma^{\prime}(s)$ is parallel to $x(\gamma(s))$ which is nothing but $\gamma(s)$. Because $\gamma^{\prime}(s)$ is a unit vector field along $\gamma$, this implies $\gamma^{\prime \prime}(s)=0$. Therefore, the integral curve $\gamma(s)=a s+b$ for some constant vectors $a$ and $b$ in $E^{n+1}$. Consequently, $\gamma^{\prime}(s)=a$ and $a s+b=f(s) a$ for some function $f$. This shows that $b$ and $a$ are parallel. Hence the integral curve $\gamma$ is a portion of the straight line which passes through the origin. Consequently, $M$ is locally a hypercone with the vertex at the origin. The converse is trivial.

Remark 4.1. Proposition 4.1 implies that open portions of straight lines are the only curves in $E^{2}$ which satisfies the condition $\langle\Delta x, x\rangle=0$.

\section{REFERENCES}

[1] B. Y. CHEN, Total mean curvature and submanifolds of finite type, World Scientific, Singapore-New Jersey-London, 1984.

[2] B.Y. CHEN, Finite type submanifolds and generalizations, University of Rome, Rome, 1985.

[3] B. Y. Chen And M. Petrovic, On spectral decomposition of immersions of finite type, Bull. Austral. Math. Soc., 44 (1991), 117-129.

[4] J. Deprez, Immersions of finite type of compact homogeneous Riemannian manifolds, Doctoral thesis, Katholieke Universiteit Leuven, 1988.

[5] F. Dillen, J. Pas and L. Verstraelen, On surfaces of finite type in Euclidean 3-space, Kodai Math. J., 13 (1990), 10-21.

[6] O. Garay, An extension of Takahashi's theorem, Geometriae Dedicata, 34(1990), 105-112.

[7] T. Hasanis and T. Vlachos, Coordinate finite type submanifolds, Geometriae Dedicata, 37 (1991), 155-165.

[8] T. TAKAHASH, Isometric immersions of Riemannian homogeneous manifolds, Tsukuba J. Math., 12 (1988), 231-233.

[9] N. Wallach, Minimal immersions of symmetric spaces into spheres, in Symmetric Spaces, pp. 1-40, M. Dekker, New York, 1972.

Department of Mathematics

Machigan STATE UNiversity

East Lansing, Michigan 48824-1027

U.S. A. 